\section{Ischemic Colitis after Hemorrhagic Shock Due to Obstetrical Reasons}

\author{
Incim Bezircioglu', Hakan Yetimalar'1, Derya Kilic Sakarya², \\ Askin Yildiz', Atilla Sencan ${ }^{3}$, Kerem Oguz ${ }^{1}$ and Burcu Kasap ${ }^{4 *}$
}

${ }^{1}$ Department of Obstetrics and Gynecology, Izmir Kâtip Çelebi University, Atatürk Training and Research Hospital, Izmir, Turkey

${ }^{2}$ Department of Obstetrics and Gynecology, Bozkır State Hospital, Konya, Turkey

${ }^{3}$ Department of Anesthesia, Izmir Kâtip Çelebi University, Atatürk Training and Research Hospital, Izmir, Turkey

${ }^{4}$ Department of Obstetrics and Gynecology, School of Medicine, Muğla Sıtkı Koçman University, Menteşe, Muğla, Turkey

\begin{abstract}
Ischemic colitis is one of the most common intestinal ischemic injuries which are difficult to diagnose and treat. In this case, we present a 28-year-old, gravid-2 para-2 woman who was diagnosed with preeclampsia and abruption of the placenta at 30 weeks of gestation as she suffered from ischemic colitis due to acute postpartum hemorrhage. To manage the ischemia supportive care as bowel rest, intravenous fluids to ensure adequate colonic perfusion and empiric broad-spectrum antibiotics to minimize bacterial translocation is performed. Ischemic colitis is a rare but potentially lethal complication of hypotension due to acute hemorrhage. It should be kept in mind during the evaluation of postpartum hemorrhage and postoperative ileus cases.
\end{abstract}

Keywords: Antepartum bleeding; Cesarean section; Gastrointestinal disorders; Preeclampsia/Eclampsia; Postpartum Hemorrhage

\section{Introduction}

Ischemic colitis is the most common form of ischemic injury of the gastrointestinal tract and arises from occlusion, vasospasm, and/or hypoperfusion of the mesenteric vessels [1]. In this case, a patient who suffered from ischemic colitis due to acute postpartum hemorrhage after cesarean section was presented.

*Corresponding author: Burcu Kasap, Department of Obstetrics and Gynecology, School of Medicine, Mugla Sıtkı Koçman University, Menteşe, Muğla 48000, Turkey, Tel: +90 2522114800/5156; E-mail: burcuharmandar@gmail.com; burcuharmandar@mu.edu.tr

Citation: Bezircioglu I, Yetimalar MH, Sakarya DK, Yildiz A, Sencan A, et al., (2015) Ischemic Colitis after Hemorrhagic Shock Due to Obstetrical Reasons. J Clin Stud Med Case Rep 2: 011.

Received: December 16, 2014; Accepted: March 13, 2015; Published: March 27, 2015

\section{Case Presentation}

A 28-year-old, gravid-2 para-2 women was diagnosed with preeclampsia and abruption of placenta at 30 weeks of gestation had been operated in emergency conditions at another hospital. Developing postpartum hemorrhage, firstly she was treated by conservative methods then transferred to our clinic in five hours. On the first examination, the patient was pale and hypotensive. Her pulse was weak and tachycardic.

Blood was oozing from the pfanenstiel's incision and there was $1500 \mathrm{ml}$ of blood in the abdominal drain. Initial blood tests showed hemoglobin to be $6.1 \mathrm{~g} / \mathrm{dl}$, leukocytes $11.200 / \mathrm{mm}^{3}$, platelets $58.500 / \mathrm{mm}^{3}$, INR 1.23, D-dimer $>4000 \mathrm{ng} / \mathrm{mL}$, aspartate transaminase $62 \mathrm{U} / \mathrm{mL}$, calcium $7.2 \mathrm{mg} / \mathrm{dL}$, and fibrinogen $166.8 \mathrm{mg} / \mathrm{dL}$. She had coagulation failure and shock.

Emergent laparotomy was performed since the patient did not respond to bimanual uterine massage and uterotonic medication. At laparotomy we found of $2 \mathrm{~L}$ hemoperitoneum. Uterine atony responded poorly to conventional uterotonics, bimanual uterine massage and also uterine artery ligation and internal iliac artery ligation. When the B-Lynch suture also failed to control bleeding, we proceeded to hysterectomy. On the postoperative first day, she developed hypertension and tachycardia (blood pressure was 230/135 $\mathrm{mmHg}$; heart rate range $136 \mathrm{bpm}$ and was treated with Esmolol Hydrochloride, Amlodipine, Furosemide and Sodium Nitroprusside. Ceftriaxon $2 \mathrm{~g} /$ day was administered. She had passage of flatus on postoperative day 2 and 3 then stabilized on postoperative day 4 .

On the fifth postoperative day, the patient complained of nausea, vomiting, abdominal pain and distension. Her bowel sounds were hypoactive and urine output was decreased. Her condition continued to deteriorate, and she developed acute renal failure, pyrexia $\left(38.4^{\circ} \mathrm{C}\right)$ and increasing distension of the abdomen rapidly. Antibiotherapy was changed as Meropenem (1000 mg $3 \times 1)$ and Teicoplanin (400 mg $1 \times 1$ ).

Abdominal examination revealed mild to moderate tenderness. In the plain abdominal radiograph, the colon was filled with gas, and the ascending colon's diameter was increased to $96 \mathrm{~mm}$ (Figure 1). Air-fluid levels were present in the dilated bowel loops on upright radiographs. Computed Tomography (CT) imaging showed widespread colonic dilatation (Figure 2). Her laboratory values were

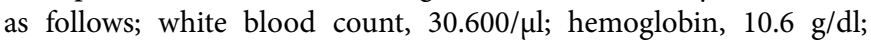
platelets, $202.000 / \mu$ l. As intestinal perforation could not be excluded, a laparotomy was performed.

At laparotomy, the whole colon, especially the ascending colon was found to be greatly distended as well as pale and ischemic. There was no evidence of mechanical obstruction, perforation or peritonitis. A rectal tube was inserted intraoperatively and external manual decompression was achieved. After the operation, oral intake was prohibited. The patient was placed on bowel rest and parenteral nutrition. Anticoagulant therapy was added. The suspicion of pseudomembranous colitis forced us to cease all antibiotics and add Metranidazole. At fifteenth day, E feacium was isolated from wound 


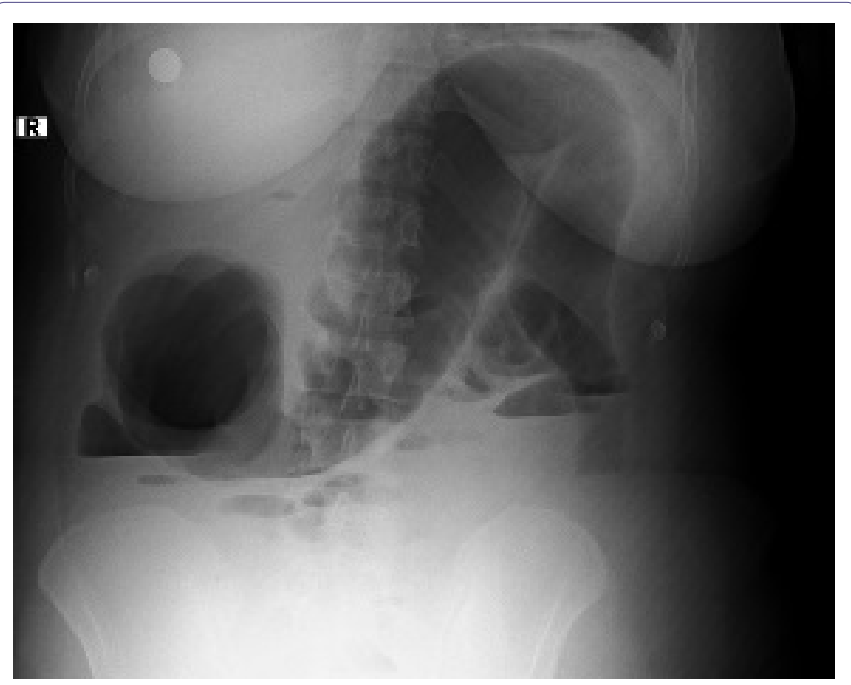

Figure 1: Air-fluid levels in the dilated bowel loops on upright radiographs.

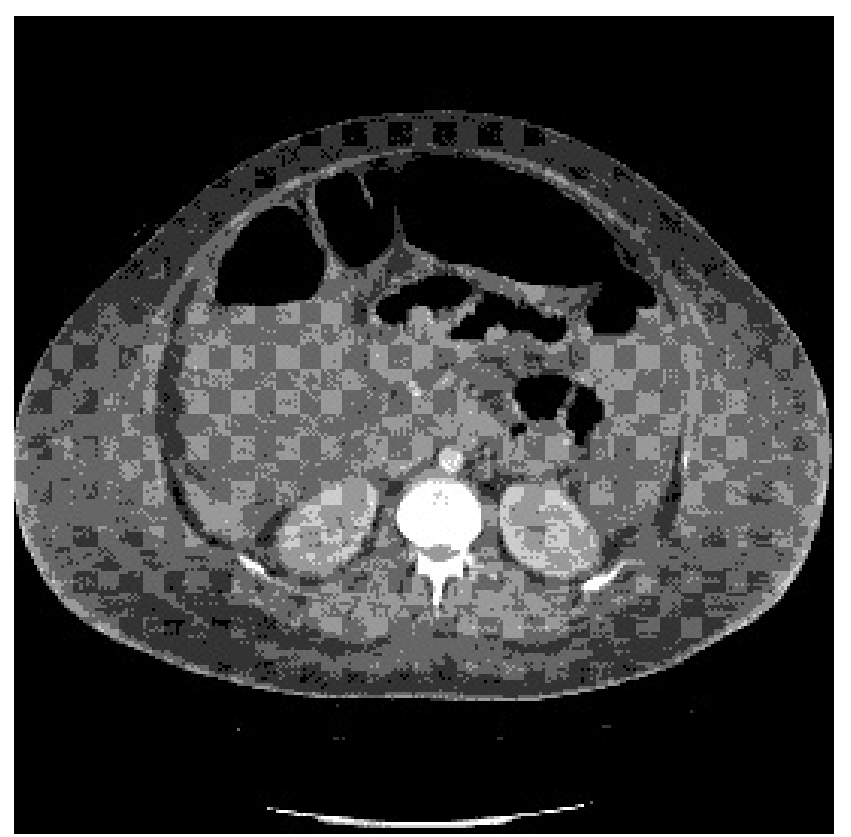

Figure 2: Dilated bowel loops on computed tomography of the abdomen.

cultures, and then coagulase negative-staphylococcus from blood cultures at eighteenth day, yeast from blood and urine cultures at twenty-third day, vancomycin-resistant $E$ feacium from stool cultures.

During this period Ciprofloxacin, Fluconozole, Anidulafungin and Piperacillin-tazobactam were administered. Since she could not tolerate enteral nutrition, total parenteral nutrition was continued until the $31^{\text {st }}$ day. During this entire period, the patient suffered from renal failure. Colonoscopy performed at the $25^{\text {th }}$ day, didn't reveal any pathological finding. Echocardiogram revealed a myopathic severely enlarged left ventricular chamber with moderate concentric left ventricular hypertrophy and severe global hypokinesis with an ejection fraction of 8 to $10 \%$. The watery stool persisted until the $38^{\text {th }}$ day and she was discharged on the $43^{\text {rd }}$ day. On her control at postoperative sixth month, she had no complaints and colonoscopy showed normal findings.

\section{Discussion}

Ischemic colitis is a commonly misdiagnosed clinical condition. It occurs from impaired mesenteric blood flow either by occlusive vascular diseases or nonocclusive diseases [1]. The vasculature of the colon is low compared with the rest of the gastrointestinal tract; it is particularly sensitive to vasospasm, and its collateral blood flow is sparse [2]. Therefore the colon is the most common site of gastrointestinal ischemia. Clinical patterns vary from transient ischemia involving the mucosa and submucosa to fulminant ischemia with gangrene. The most common mechanisms that can cause colonic ischemia are hypotension and hypovolemia in connection with sepsis, impaired left ventricular function, dehydration or hemorrhage producing a compromise in systemic perfusion and triggering a reflex mesenteric vasoconstriction. In our patient, hemorrhagic shock induced hypoperfusion which caused mesenteric ischemia. The state of increased coagulability due to pregnancy and the increase of peripheral vascular resistance due to preeclampsia probably contributed to the pathogenesis of ischemic colitis.

As in our case, ischemic colitis usually presents with acute-onset abdominal pain and cramping. Other usual symptoms are diarrhea and/or hematochezia (68\%), abdominal distention (63\%), and nausea/vomiting (38\%) $[3,4]$. Clinical examination often reveals just abdominal tenderness. Peritoneal signs correspond to more advanced disease like transmural necrosis and it should be always kept in mind that systemic signs of SIRS (Systemic Inflammatory Response Syndrome) may develop [3,4]. Plain abdominal radiographs are generally insensitive and they often show generalized bowel distension and air-filled bowel loops as in our patient. And the most common finding of CT in the evaluation of ischemic colitis is segmental circumferential wall thickening [5].

In the absence of colonic gangrene or perforation, to manage the ischemia supportive care as bowel rest and intravenous fluids to ensure adequate colonic perfusion is performed. Empiric broad-spectrum antibiotics are often administered in patients with moderate to severe colitis to minimize bacterial translocation and sepsis. Careful monitoring is necessary for signs of necrosis, such as persistent fever, leukocytosis, peritoneal irritation, or protracted pain or bleeding [6].

In our patient, significant fluid leakage occurred to the extra vascular space because of the edema due to preeclampsia, in the form of watery diarrhea with abundant liquid passage from damaged intestinal epithelium to the lumen and polyuria secondary to pre-renal type renal failure.

Ischemic colitis is reversible in about half of the cases. Two thirds of patients with reversible disease will clinically improve within 24 to 48 hours, and resolving of all endoscopic and radiologic abnormalities may take 1 to 6 months $[2,6]$. In the presented case, the medications used for intractable hypertension, tachycardia, hemodynamic instability and nasocomial infections decayed the healing process. And it resolved with a conservative treatment.

In conclusion, ischemic colitis is a rare but potentially lethal complication of hypotension due to hemorrhage. It is difficult to diagnose especially in young woman and difficult to treat. It should be kept in mind during the evaluation of postpartum hemorrhage and postoperative ileus cases. 
Citation: Bezircioglu I, Yetimalar MH, Sakarya DK, Yildiz A, Sencan A, et al., (2015) Ischemic Colitis after Hemorrhagic Shock Due to Obstetrical Reasons. J Clin Stud Med Case Rep 2: 011.

- Page 3 of 3 •

\section{References}

1. Higgins PD, Davis KJ, Laine L (2004) Systematic review: the epidemiology of ischaemic colitis. Aliment Pharmacol Ther 19: 729-738.

2. Green BT, Tendler DA (2005) Ischemic colitis: a clinical review. South Med J 98: 217-222

3. Zou X, Cao J, Yao Y, Liu W, Chen L (2009) Endoscopic findings and clinicopathologic characteristics of ischemic colitis: a report of 85 cases. Dig Dis Sci 54: 2009-2015.
4. Washington C, Carmichael JC (2012) Management of ischemic colitis. Clin Colon Rectal Surg 25: 228-235.

5. Thoeni RF, Cello JP (2006) CT imaging of colitis. Radiology 240: 623-638.

6. Baixauli J, Kiran RP, Delaney CP (2003) Investigation and management of ischemic colitis. Cleve Clin J Med 70: 920-921, 925-926, 928-930. 